\title{
Gamification as an instrument for organizational behaviour change during the meeting: case study «ROBATIEMPOS»
}

\author{
Iana DULSKAIA \\ Sapienza University of Rome, Rome, Italy \\ Francesco BELLINI \\ Sapienza University of Rome, Rome, Italy \\ francesco.bellini@uniroma1.it \\ Roman ZABAL \\ Cookie Box s.r.l, Barcelona, Spain \\ Oscar GARCIA PAÑELLA \\ ENTI-UB Barcelona, Spain \\ Fabrizio D'ASCENZO \\ Sapienza University of Rome, Rome, Italy
}

\begin{abstract}
During the last years, gamification has been a burning issue and a subject of scientific discussions. Gamification is an innovative solution to foster motivation. It relates to the use of game elements in non-game contexts. By applying game mechanics to training and other business critical processes, organizations can improve employee engagement, boost productivity and lift operational performance. Over the last few decades, it has been noticed the significant enhancement of time spent attending meetings. Meetings are essential for the organizations, they improve the effectiveness of a company team working and are necessary for human relations. Nevertheless, in many cases meetings are considered as a waste of time. There are very few scientific papers that regarding the use of gamification for business meeting changes. The study seeks to explore the behavioural change of participants towards business meetings after introducing a business game, in order to come up with an interesting solution that might be useful for other researchers and practitioners in understanding the use of gamification in similar contexts. With regard to this aim, a single-case holistic design methodology is used and qualitative approach using semi-structured interviews with gamification experts and project managers is applied. This paper contributes both to the scientific literature and to managerial implication.
\end{abstract}

Keywords: gamification, cultural change, business meetings, meeting efficiency.

\section{Introduction}

During the last years, gamification (Deterding et al.,) has been a burning issue and a subject of scientific discussions.

Gamification is an innovative solution to foster motivation. It relates to the use of game elements in non-game contexts (Deterding et al., 2011). Scholars highlight that recently gamification is applied within the organisations in such fields like marketing, performance management, sales management, human resources (Muntean, 2011) as well as non-business contexts such as politics, health (Lee \& Hammer, 2011, interactive systems and also education (Lee \& Hammer, 2011). Global researcher Markets and Markets predicts 
that gamification will be worth $\$ 5.5$ billion by 2018 , compared with its present size of $\$ 421$ million.1

Gamification uses game design elements to solve organizational problems in obtaining companies' goals effectively through user engagement. The users can be stakeholders from inside the organization, consumers of products or services, individuals or groups (Gartner, 2012).

PICBE | 360

By applying game mechanics to training and other business critical processes (Crombie et al., 2016) organizations can improve employee engagement, boost productivity and lift operational performance. There are different reasons why organisations can decide to change employees' behaviour. It depends on the problems that organization is facing or goals that it is setting. One of such business challenges is inefficient meetings conduction. Over the last few decades it has being noticed the significant enhancement of time spent attending meetings.

Meetings are essential for the organisations (Rogelberg et al., 2006) they improve the effectiveness of a company team working and are necessary for human relations. Nevertheless, in many cases meetings are considered as a waste of time. Experts in meeting management and business productivity came up to the idea that unproductive and insufficient business meetings can cause great costs for any type of organization (Brett et al., 2006). Bang et al., (2010) have stated in their research that third of business meeting time is unproductive and bring to the enhancement of costs. Due to the financial costs enhancement organisations are trying to improve the effectiveness of business meetings.

Following the problem statement, the behavioural change in the business meetings and the application of such techniques will be described in this work.

\section{Literature review \\ Gamification phenomenon}

In the scientific literature about gamification there are multiple concepts and definitions that are used to identify how games are used outside the "traditional" game environment (McGonical, 2011).

In the Table 1 below some of the gamification's definitions are presented.

Table 1. Gamification Definition

\begin{tabular}{|l|l|}
\hline Definition of Gamification & Author \\
\hline $\begin{array}{l}\text { A process of enhancing services with (motivational) } \\
\text { affordances in order to invoke gameful experiences } \\
\text { and further behavioural outcomes }\end{array}$ & $\begin{array}{l}\text { (Huotari \& Hamari, 2012), } \\
\text { (Hamari, 2013) }\end{array}$ \\
\hline $\begin{array}{l}\text { The use of game design elements in non-game } \\
\text { contexts }\end{array}$ & (Deterding et al, 2011) \\
\hline $\begin{array}{l}\text { A process of adding game mechanics to process, } \\
\text { programs and platforms that wouldn't traditionally } \\
\text { use such concepts. }\end{array}$ & (Swan, 2012) \\
\hline $\begin{array}{l}\text { Adaptation and application of game design principles } \\
\text { and game interaction elements to workplace } \\
\text { processes and behaviours }\end{array}$ & (Oprescu et al., 2014) \\
\hline
\end{tabular}

Source: Authors'.

However, the use of game thinking in business is not new, gamification is still considered as a growing process that is gaining more and more attention. The figure below 
represents a Gamification position according to Gartner technology hype cycle 2014. It can be noticed that Gamification will reach the plateau only in the next 5- 10 years.

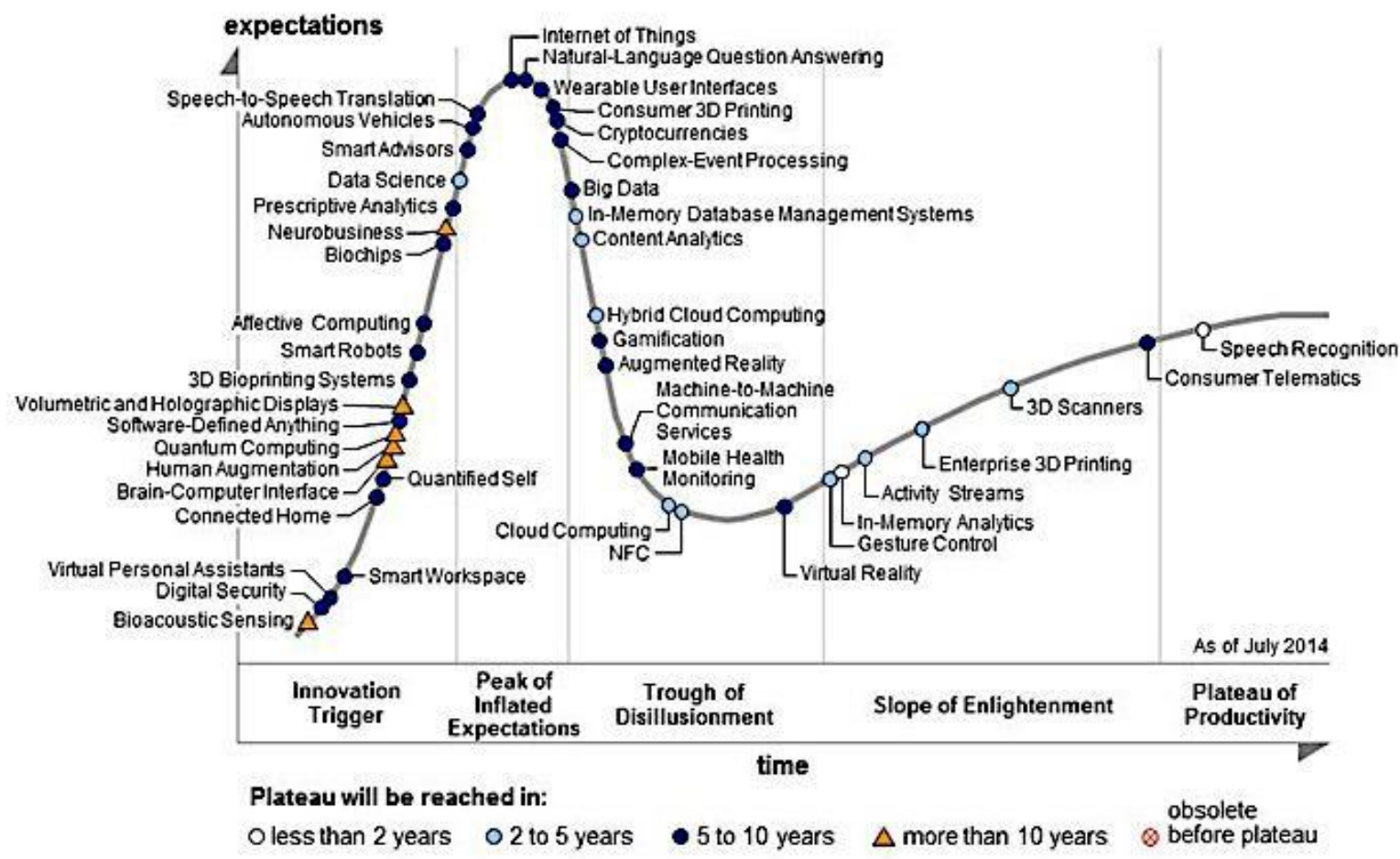

PICBE | 361

Figure 1. Gartner Technology hype cycle 2014

Source: http://www.gartner.com/newsroom/id/2819918.

Gamification is the use of game design (Deterding et al, 2011) and game mechanics (Swan, 2012) to engage a target audience to change behaviour, learn new skills or engage in innovation (Gartner, 2012). The audience can be customers, employees or the general public (Singh, 2012), however first of all, they are people with needs and desires who will respond to stimulus. In this case It is crucial to think that people in these target audiences are the "players" of gamified applications.

Gamification uses the same design techniques and game mechanics found in the games, but it applies them in non-game contexts (Deterding et al., 2011) including: customer engagement, employee performance, training and education, innovation management, personal development, sustainability and health. According to Gartner (2012) all areas of business could benefit from gamification because it can help to achieve three broad business goals: to change behaviour; to develop skills; to enable innovation.

In order to summarize the phenomenon of gamification we would like to address to Deterding et al (2011) that highlighted that "Gamification" refers to

- the use (rather than the extension) of

- design (rather than game-based technology or other game related practices)

- elements (rather than full-fledged games)

- characteristic for games (rather than play or playfulness)

- in non-game contexts (regardless of specific usage intentions, contexts, or media of implementation). 


\section{Gamification theories}

MDA framework as it gives an in-depth insight to the mechanisms that lies in basis of gamification. MDA stands for Mechanics, Dynamics, and Aesthetics.

This approach was created by Hunicke, LeBlanc and Zubek, in 2004 and has being presented and taught as part of the Game Design and Tuning Workshop at the Game Developers Conference, San Jose 2001-2004. It has its aim to provide a better understanding to a game design. The MDA approach divides a game design in 3 main components (see Figure 2). Each of components is described below in more details.

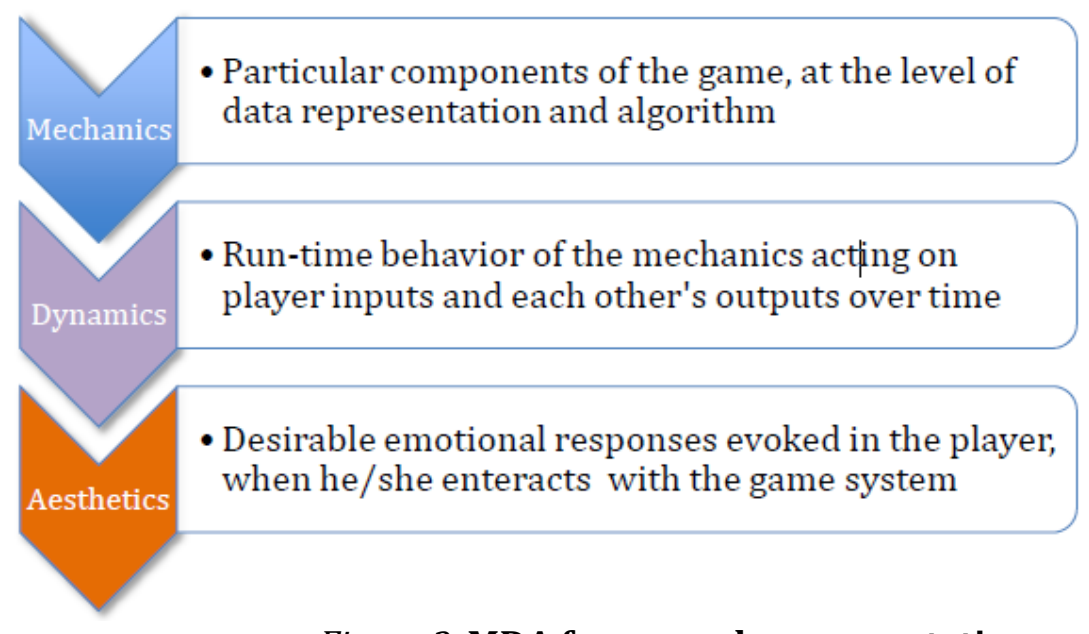

Figure 2. MDA framework representation

Source: Hunicke et al., 2004.

Mechanics: this component includes different actions, missions, behaviours and control mechanisms that the user can apply within a game context. Together with the game's content the mechanics support overall gameplay dynamics (Hunicke et al., 2004)

Dynamics: after the mechanics provide a start to dynamic system behaviour, the user begins to develop a certain aesthetic experiences by applying dynamic techniques as time pressure and opponent play as in challenge kind of game or providing information across game members as in example of fellowship game.

Aesthetics: represents emotional attitude of a player regarding the game. The authors (Hunicke et al., 2004) provided a taxonomy of the elements that can create emotional aspects of the game.

Another important theory in which gamification finds its basis is Self-Determination Theory (SDT) describes social-contextual conditions that facilitate to predict motivational processes (Sailer, 2014). SDT focuses on three main psychological needs: competence (ability to produce desired outcomes and to experience mastery and effectiveness), autonomy (the ownership of one's behaviour) and social relatedness (feeling of being connected with others) (Ryan and Deci, 2000). SDT theory focuses on six classifications of distinct types of motivation: intrinsic (autonomous motivation), motivation and four extrinsically (controlled motivation) motivated behaviours: external regulation, introjected regulation, identified regulation and integrated regulation (Ryan and Deci, 2000), each of which has specific consequences for learning, performance, personal experience, and wellbeing. Intrinsic motivation involves people doing an activity because they find it interesting, challenging or it has an exciting nature (Deci and Ryan, 1985). It brings spontaneous satisfaction from the activity itself, the opposite is extrinsic motivation that requires an 
instrumentality between the activity and some separable consequences such as tangible rewards, so satisfaction comes not from the activity itself but rather from the extrinsic consequences to which the activity leads (Porter and Lawler, 1968). Psychologists that work on the themes of organizational behaviour have distinguish the significance of intrinsic motivation in work organizations and have also noted the important role of tangible extrinsic rewards for motivating behaviour. Another conceptual model of gamification effectiveness was proposed by Kappen and Nacke (2013) called «Kaleidoscope of Effective Gamification» where they described the main instruments that should be taken into consideration in order to elaborate effective gamification within the organisation. Figure 5 demonstrates the model of gamification effectiveness.

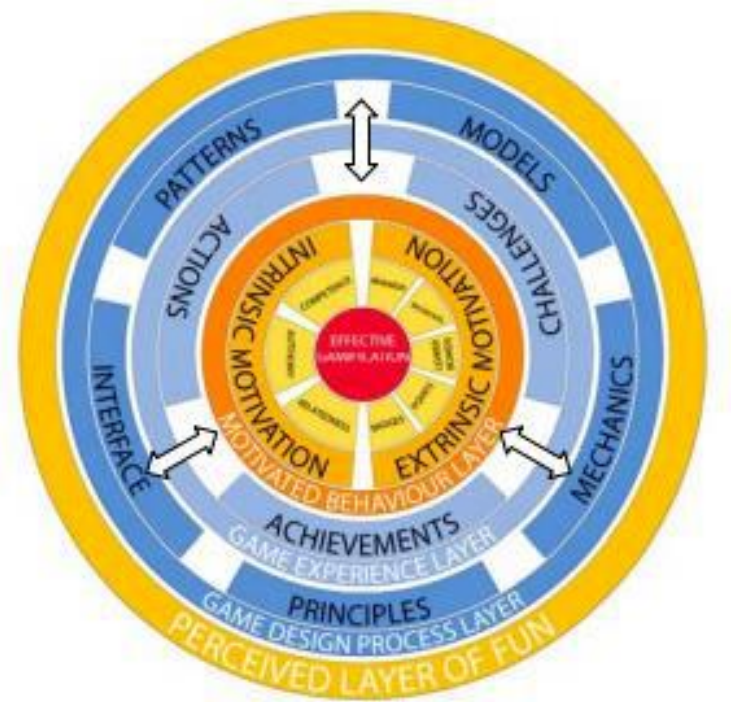

Figure3. - Kaleidoscope of Effective Gamification

Source: Kappen and Nacke, 2013.

1. Effective Gamification Core. In the central core of the kaleidoscope is established the nucleus of player experience, which is the alliance of all model layers. It includes core objectives of a design enabling effective gamification.

2. Motivated Behaviour Layer. An important layer in which it has to be identified a user need that bases on intrinsic and extrinsic motivation. The intrinsic motivation in this layer is composed of three main needs: competence, autonomy and relatedness. The extrinsic motivation part of this layer consists of badges, points, leader boards and incentives and rewards. It is crucial to choose right motivations in order to stimulate players to reach the goal of the game. After the motivation for the desired behaviour was chosen the next layer to be addressed is a Game Experience Layer.

3. Game Experience Layer. In order to create a gameplay experience with intrinsic and extrinsic motivational stimulus as a focus, it is essential to integrate actions, challenges and achievements in the gamification design process. This can permit the development of an engaging gameplay experience within a "gamified" application. The experience of a user created in this layer is connect with the next outer ring called the Game Design Process Layer.

4. Game Design Process Layer: By integrating the game elements for creating a fun experience for the player it becomes necessary to identify this layer as a process. Game design principles, mechanics, models, patterns and interface design elements needed as 
related subsystems permitting this layer to function since they were created to pass the Game Experience Layer.

5. Perceived Layer of Fun. This layer has an important connection with Motivated Behaviour Layer. Intrinsic motivation can be a great stimulus to influence a player to participate in a game. Perceived Layer of Fun is what a player can aesthetically experience in terms of audio, visuals, interface design, tangible interactions and intangible experiences. These experiences appear during gameplay through actions, challenges and achievements that engage the player.

\section{Gamification inside the organisation}

According to the author (McCormick, 2013;) the main goals of using gamification within the organisation are to enhance intrinsic employee motivation and productivity in order to obtain a desired behaviour.

Gamification uses motivational factors based on needs and desires to get organizational task completed. Organisational task together with game engagement and actions can make people to be excited about work and boost productivity (Wu 2011).

Gamification through its power to communicate goals and provide real time feedback about employee achievement is a perfect organizational behavioural change tool, enabling structural change. Gamification is a crucial tool of transformational change. It can be used to better support user engagement and feedback, giving powerful indicators of process improvement. For instance, to change work habits, gamification can provide an explanation of the change, through gamified e-learning, support the change, form habits and drive engagement (Gartner, 2012).

\section{Case study: Robatiempos}

Due to the privacy issue the company name is not mentioned in this work (for facilitating reasons it will have a name "Company X")

Company $\mathrm{X}$ is a Spanish international company in the field of electrical equipment. The project started in September 2015 by the request of "Company X" to help to change the company culture in relation to meetings and time management.

The project had the duration of 5 months (September 2015 - January 2016). Cookiebox is a company that organized the Robatiempos project in order make Company's X meeting more efficient.

In order to give more structured information about the project it was decided to use Gamification use case canvas. A "gamified" essential use case (Gears and Braun, 2013) specified goals, objectives, beneficiaries, business rules, behavioural norms, preconditions, actors, and system interactions. The use case provided a canvas to articulate business rules that could not be broken; personal, social, and corporate norms that could be challenged; and conditions necessary for a successful outcome.

Table 2. Gamification use case canvas

\begin{tabular}{|l|c|}
\hline \multicolumn{2}{|c|}{ Gamification use case canvas } \\
\hline $\begin{array}{l}\text { Goals (primary purposes of } \\
\text { the experience) }\end{array}$ & $\begin{array}{l}\text { - Improve the problem } \\
\end{array}$ \\
\hline Objectives: (derivable & Optimulate interest, increase communication, reduce frustration \\
\hline
\end{tabular}




\begin{tabular}{|l|l|}
\hline $\begin{array}{l}\text { accomplishments offered in } \\
\text { the experience) }\end{array}$ & $\begin{array}{l}\text { company, to improve the management of internal meetings and make } \\
\text { them more efficient. }\end{array}$ \\
\hline $\begin{array}{l}\text { Business Rules (constrains } \\
\text { or policy that cannot be } \\
\text { broken) }\end{array}$ & Players have to follow the rules of the games \\
\hline $\begin{array}{l}\text { Behavioural norms } \\
\text { (personal, social, and } \\
\text { corporate norms that can be } \\
\text { challenge) }\end{array}$ & $\begin{array}{l}\text { Behaviour of the employees during the business meetings (negative or } \\
\text { positive) }\end{array}$ \\
\hline $\begin{array}{l}\text { Preconditions } \\
\text { (circumstances the domain } \\
\text { that must be true before } \\
\text { interactions to enable } \\
\text { positive outcome) }\end{array}$ & $\begin{array}{l}\text { Meeting behaviour of the employees that was written down in the } \\
\text { Decalogue }\end{array}$ \\
\hline $\begin{array}{l}\text { Actor (performers involved } \\
\text { in the problem domain) }\end{array}$ & $\begin{array}{l}\text { Board of Directors } \\
\text { Middle managers }\end{array}$ \\
\hline $\begin{array}{l}\text { Normal course of action } \\
\text { gameful interactions } \\
\text { specified in framework) }\end{array}$ & Robatiempos game creation for meeting improvement \\
\hline
\end{tabular}

PICBE | 365

Source: Gears and Braun, 2013.

Cookie Box proposed a co-creation game-based work with Board of Directors and managers in order to develop together the new meeting "ritual" and materials to make change effective. These elements were audio video pieces to introduce narrative and business dysfunctions created during the meetings, a Decalogue of appropriate behaviours and a game of cards to combat inefficient behaviour in a fun manner. The scope was to raise awareness among all of the techniques of efficient use of time in meetings and to create a physical element that constantly remembers these techniques of efficiency and their antagonists, Robatiempos (Timestealers).

In order to obtain this result, it was decided to provide 3 Workshops (duration of each was 4 hours).

The audience of the workshop was:

- Board of Directors

- Middle Managers

- More than 40 people from "Company X" took part in the Workshops.

The lectures were given by Román Zabal Bustamante, Cookie box expert in Storytelling, Gamification, Innovation \& Time Management.

\section{Conclusion}

To conclude, the project was an extraordinary example of the usage of fusion of Storytelling, Gamification and Co-creation. The workshops provided the game-based elements that have allowed organizational behavioural changes to occur. The workers themselves have created elements that have been brought to the reality of the company, with their dysfunctions and real stories as the main input. Game of cards helped to change the company culture in relation to meetings and time management.

As the result it was demonstrated a high tracking and feedback of the Robotiempos newsletters campaign; positive feedback on this character; and extensive diffusion and awareness of the new effective process for providing meeting: Robatiempos is already part of Company $\mathrm{X}$ vocabulary and culture. 
Feedback that was given after the workshops by the participants:

"Very interesting approach, dynamic and keen on receiving the different materials to help them change those dysfunctional aspects" (the cards for the game of Cards that the participants created during the Workshops and the Robatiempos -Timestealer- AV campaign pieces with advices to become a master in wasting your colleagues' time, created with their company real stories).

This paper can contribute to the scientific literature as there is a gap in the literature regarding the use of gamification for the organizational behaviour. It can be also useful for the for the managers and policy makers as the Robatiempos project teaches how to provide a wise time management. The next step will be to provide the interview with managers participated in the workshops in order to understand if the companies' meeting became more efficient.

\section{References}

Bang, H., Fuglesang S. L., Ovesen, M. R. \& Eilertsen D. E. (2010). Effectiveness in Top Management Group Meetings: The Role of Goal Clarity, Focused Communication, and Learning Behavior. The Scandinavian Journal of Psychology, 1-9.

Brett, H., Brett, G., \& Strauch, M. (2006). U.S. Patent Application No. 11/556,420.

Crombie D., Mersch P., Dulskaia, I. \& BELLINI F., (forth.) 2016. The JamToday Network: the European Learning Hub for Applied-Games for Learning Environments, in "Digital technology and organizational change: Reshaping technology, people, and organizations towards a global society" - LECTURE NOTES IN INFORMATION SYSTEMS AND ORGANISATION (LNISO), Springer (R)

Deci, E. L., \& Ryan, R. (1985). Intrinsic motivation and self-determination in human behaviorPlenum Press. New York.

Deterding, S., Dixon, D., Khaled, R., \& Nacke, L. (2011, September). From game design elements to gamefulness: defining gamification. In Proceedings of the 15th international academic MindTrek conference: Envisioning future media environments (pp. 9-15). ACM.

Gartner (2012) Gamification: engagement strategies for business and IT.

Hamari, J. (2013). Transforming homo economicus into homo ludens: A field experiment on gamification in a utilitarian peer-to-peer trading service. Electronic commerce research and applications, $12(4), 236-245$.

Hunicke, R., LeBlanc, M., \& Zubek, R. (2004, July). MDA: A formal approach to game design and game research. In Proceedings of the AAAI Workshop on Challenges in Game AI (Vol. 4, p. 1).

Huotari, K., \& Hamari, J. (2012, October). Defining gamification: a service marketing perspective. In Proceeding of the 16th International Academic MindTrek Conference (pp. 17-22). ACM.

Kappen, D. L., \& Nacke, L. E. (2013). The kaleidoscope of effective gamification: deconstructing gamification in business applications. In Proceedings of the First International Conference on Gameful Design, Research, and Applications (pp. 119122). ACM.

Lee, J. J., \& Hammer, J. (2011). Gamifi cation in education: What, how, why bother? Academic Exchange Quarterly, 15 (2).

McCormick, T. (2013). Anthropology of an idea gamification. Foreign Policy, (201), 26. 
McGonigal, J. (2011). Reality is broken: Why games make us better and how they can change the world. Penguin.

Muntean, C. I. (2011). Raising engagement in e-learning through gamification. In Proc. 6th International Conference on Virtual Learning ICVL (pp. 323-329).

Oprescu, F., Jones, C., \& Katsikitis, M. (2014). I PLAY AT WORK-ten principles for transforming work processes through gamification. Frontiers in psychology, 5.

PICBE | 367

Porter, L. W., \& Lawler, E. E. III. (1968). Managerial attitudes and performance. Homewood, IL: Irwin-Dorsey.

Rogelberg, S. G., Burnfield, J. L, Leach, D. J. \& Warr, P. B. (2006). “Not Another Meeting!” Are Meeting Time Demands Related to Employee Well-Being?. Journal of Applied Psychology, 91 (1). 83-86.

Ryan, R. M., \& Deci, E. L. (2000). Self-determination theory and the facilitation of intrinsic motivation, social development, and well-being. American psychologist, 55(1), 68.

Sailer, M., Hense, J., Mandl, H., \& Klevers, M. (2013). Psychological Perspectives on Motivation through Gamification. IxD\&A, 19, 28-37.

Swan, C. (2012). Gamification: A new way to shape behavior. Communication World, 29(3), 13-14.

Wu, M. (2011). Gamification from a company of pro gamers. Lithosphere Community. 